\title{
Regulatory T Cell Adaptation in the Intestine and Skin
}

Natasha Whibley ${ }^{1}$, Andrea Tucci ${ }^{1}$, Fiona Powrie ${ }^{1,2}$

Figure 1. Treg development and subsets in the intestine and skin.

A) Two developmentally distinct Treg subsets are recognised. Thymic-derived Tregs (tTregs) differentiate into Foxp3 ${ }^{+}$Tregs in the thymus whereas peripherally induced Tregs (pTregs) leave the thymus as naïve $C D 4^{+} \mathrm{T}$ cells and differentiate into Tregs in secondary lymphoid organs (SLOs) following $T$ cell receptor (TCR) stimulation and differentiation signals. It is believed that upon activation, tTregs and pTregs upregulate expression of activation markers and tissue-homing receptors in response to environmental cues that drive their migration to intestinal and skin sites.

B) The small intestine, colon and skin are distinct environments with unique physiological functions (bullet points). Three major Treg subsets are recognised in the intestine and skin, namely ROR $\gamma \mathrm{t}^{-}$pTregs, ROR $\gamma \mathrm{t}^{+}$pTregs and GATA3 ${ }^{+}$tTregs. Although pTregs and tTregs are present in both the intestine and skin, Treg subset composition is unique between intestinal and skin sites. ROR $\gamma \mathrm{t}^{-}$and $\mathrm{ROR} \gamma \mathrm{t}^{+} \mathrm{pTregs}$ are present at similar frequencies in the small intestine, whereas $R O R \gamma t^{+}$pTregs dominate the colonic $p$ Treg pool. GATA3 ${ }^{+}$tTregs comprise a minor fraction of the small intestinal and colonic Treg pool. In contrast, the skin Treg pool is almost exclusively composed of GATA3 ${ }^{+}$tTregs. A minor pTreg population is present in the skin, although its phenotype is unknown. The relative size of each Treg subset cell relates to the relative frequency within the total Treg pool in the intestine and skin.

\section{Figure 2. Host and environmental pathways that shape intestinal Tregs.}

Intestinal Treg subsets are shaped by different host and environmental factors and perform distinct functions (boxes). ROR $\mathrm{t}^{+}$and ROR $\mathrm{Rt}$ p $\mathrm{p}$ Treg maintenance depends on the microbiota. However, the microbiota has a stronger impact on ROR $\gamma \mathrm{t}^{+} \mathrm{pTregs}$ whereas ROR $\mathrm{t}^{-}$pTreg maintenance is strongly affected by dietary antigens (Ags). Short-chain fatty acids (SCFAs) derived from the microbiota may shape both pTregs and tTregs whilst evidence suggests that aryl hydrocarbon receptor (AhR) ligands shape $p$ Tregs only. The vitamin A metabolite, retinoic acid (RA), is generated by retinal dehydrogenase (RALDH) activity in $\mathrm{CD} 103^{+}$dendritic cells (DCs) and promotes ROR $\gamma \mathrm{t}^{+}$ pTregs. RA likely acts in concert with TGF- $\beta 1$ and IL-6 for optimal ROR $\mathrm{t}^{+}$pTreg differentiation. The cytokines that promote ROR $\gamma \mathrm{t}^{-} \mathrm{pTreg}$ are unknown, but may include 
TGF- $\beta 1$ and IL-2. IL-2 is required for GATA3 ${ }^{+}$tTreg maintenance. IL-33, produced in response to tissue damage, may also be central to intestinal GATA3 ${ }^{+}$tTreg responses. Common features of intestinal Tregs include expression of receptors required for survival, such as TNFR2, molecules associated with suppressive function, such as IL10 , and the gut homing receptors, CCR9 and $\alpha 4 \beta 7$. Dashed arrows indicate that further evidence is needed to be conclusive. Red arrows indicate TCR specificity of the Treg subsets.

\section{Figure 3. Treg functions in the intestine and skin.}

Tregs in the intestine and skin perform shared functions, such as control of aberrant inflammatory responses. However, Tregs also perform unique tissue-specific functions, demonstrating adaptation to intestinal and skin sites.

\section{Figure 4. Host and environmental pathways that shape skin Tregs}

The skin Treg pool is dominated by GATA3 ${ }^{+}$tTregs, with pTregs comprising a minor fraction. Ultraviolet $B(U V B)$ radiation is a strong promoter of skin tTregs. Although the in vivo mechanism is unknown, UVB metabolises 7-dehydrolcholesterol (7-DHC) to the bioactive form of vitamin $\mathrm{D}, 1,25\left(\mathrm{OH}_{2}\right) \mathrm{D}_{3}$ (vitamin $\mathrm{D} 3$ ), which can signal through the vitamin $\mathrm{D}$ receptor (VDR) to directly or indirectly promote tTregs in vitro. Vitamin A, through metabolism to its active form retinoic acid (RA) by $\mathrm{RALDH}^{+}$skin dendritic cells (DCs), may induce pTregs in the skin. The microbiota may also be involved in shaping skin Treg subsets either directly or through production of metabolites such as short-chain fatty acids (SCFAs) or aryl hydrocarbon receptor (AhR) ligands. The host cytokines that shape skin Tregs are unknown, but IL-33 perhaps produced following damage, and IL-2, IL-7, TGF- $\beta 1$ and TSLP have been implicated. Overall, very little is known about the host and environmental pathways that regulate skin tTregs and pTregs, including Treg antigen specificity. Dashed arrows indicate that further evidence is needed to be conclusive. Red arrows indicate TCR specificity of the Treg subsets.

\section{Figure 5. Model of Treg metabolic adaptation at barrier sites.}

The metabolic state of a cell is highly dependent on the local availability of metabolic substrates, such as glucose, amino acids (AAs) and lipids, as well as physiological factors such as $\mathrm{O}_{2}, \mathrm{pH}$ and temperature. Little is known about metabolic adaptation of Tregs at barrier sites, but evidence suggests that glycolysis is associated with Treg activation and secondary lymphoid organ (SLO) environments that are rich in glucose 
and glutamine. In contrast, oxidative phosphorylation (OXPHOS) and fatty acid metabolism are associated with Tregs in lipid-rich environments like the intestine and skin and $T$ cell homeostasis and survival at these sites. Foxp3 regulates metabolic pathways associated with protection from cellular stress in vitro, and therefore may endow Tregs with a fitness advantage at sites of high stress, such as barrier sites. Overall, Treg metabolic adaptation at barrier sites is likely to be strongly linked to Treg phenotype and function. 